\title{
Improving Learning Electrical Engineering Student Activity via Computer Simulation
}

\author{
Hanalde Andre ${ }^{1 *}$ Amirul Luthfi ${ }^{1}$ Baharuddin ${ }^{1}$ Rizki Wahyu Pratama ${ }^{1}$ \\ ${ }^{1}$ Department of Electrical Engineering, Universitas Andalas, Padang, Sumatera Barat 25175, Indonesia \\ *Corresponding author. Email: hanalde.andre@eng.unand.ac.id
}

\begin{abstract}
Student activity in learning is an important part of achieving outcome course. This research concerns about improving learning student activity via computer simulation in the academic year 2019-2020. In general, the learning media here is anything that can be used to stimulate the thoughts, feelings, attention, and abilities of learners to encourage the learning process. The development of learning technology that is carried out is related to the use of software to help students understand Telecommunication Electromagnetic courses. Evaluation of the use of learning media with computer simulations is carried out using an online system. all students who take this lecture are 25 students. Computer simulations designed to improve student learning activities are well accepted. based on student responses to learning activities that are preferred, the use of computer simulations is $52 \%$, then group discussions, assignments and lectures are $24 \%, 16 \%$ and $8 \%$ respectively
\end{abstract}

Keywords: Learning Student Activity, Computer Simulation, Style Learning

\section{INTRODUCTION}

Learning process requires media as a tool used to convey learning messages. In general, the learning media here is anything that can be used to stimulate the thoughts, feelings, attention and abilities or skills of learners so that they can encourage the learning process. Discovery Learning and Small Group Discussion to increase students' ability to understand the course material [1-2]. visual interactive learning media can improve student learning outcomes on the topic of the human motion system [3-4]. motivation is an important part of the learning process so that it can increase the expected achievements in a lecture [5].

Learning outcomes of the Telecommunication Electromagnetic course:

a. Able to calculate wave propagation parameters (speed, wavelength, electric field, magnetic field, propagation constant) in the medium.

b. Able to analyse and compare wave propagation in various mediums. c. Able to calculate the uniform wave power in the medium (free space, dielectric, conductor).

d. Able to calculate the reflection coefficient and transmission coefficient

e. Able to calculate Standing Wave (SWR)

f. Able to model a transmission line as an electrical circuit

g. Able to calculate transmission line parameters at low frequency and high frequency

h. Able to use the graph method (Smith Chart) to solve problems on the transmission line

i. Able to communicate effectively

The learning methods applied so far are still using the Teaching Centre Learning (TCL) method. At the beginning of the lecture, the lecturer explains the material that will be obtained during the lecture which is outlined through SAP / RPKPS and lecture contracts. Each lecturer meeting explains the lecture material, is given the opportunity to ask questions, tries to do exercises, quizzes and the end of the lecture is given an 
assignment. In Figure 1, you can see the grading distribution of the course for the 2018/2019 academic year.

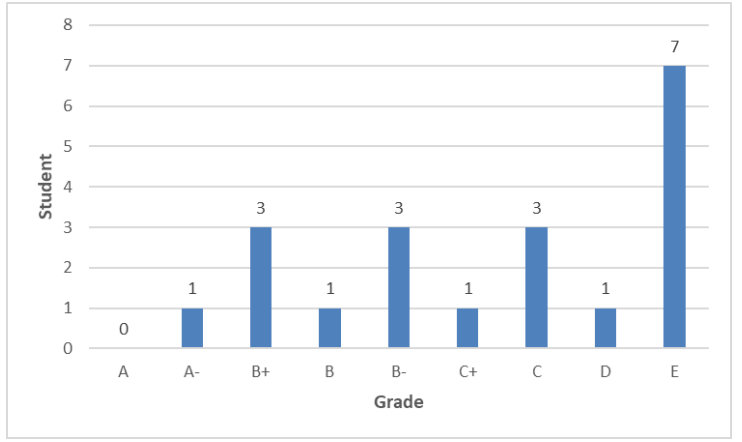

Figure 1. Grade of Telecommunication

Electromagnetic Course for the 2018/2019

Academic Year

The learning technology that will be applied in the Telecommunication Electromagnetic course uses a simulator in the form of software. The material in this lecture is the application of mathematics in explaining the phenomenon of electromagnetic waves in telecommunications. The learning technology that will be applied is expected to be able to build a student's frame of mind on the physical events that occur and analyse them in mathematical equations.

Use of MATLAB software to show simulations of electromagnetic wave applications in telecommunications. MATLAB (Matrix Laboratory) is a numerical computing environment and fourth generation computer programming language. Developed by The MathWorks, MATLAB allows manipulation of matrices, plotting of functions and data, implementing algorithms, creating user interfaces, and interfacing with programs in other languages. Although only numerical in nuance, a toolbox that uses the MuPAD symbolic engine, allows access to computer algebra capabilities. An add-on package, Simulink, adds multichannel graphic simulation and Model-driven Design for embedded and dynamic systems

\section{METHODS}

The development of learning technology that iscarried out is related to the use of software (software) to help students understand Telecommunication Electromagnetic courses. Software created using MATLAB. The learning application designed in this study is the transmission line characteristic calculation application. A transmission line's characteristics are one of the important materials in telecommunications, where these characteristics are used to calculate the power of the electromagnetic waves reaching the receiver. Electromagnetic waves' power is significant for determining the type of modulation, antenna specifications, and specifications of the amplifier used in the telecommunications process.

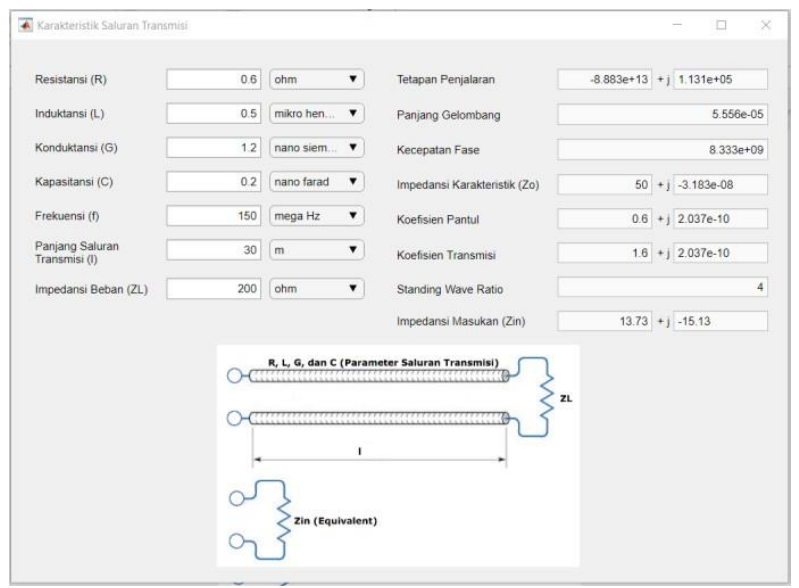

Figure 1. Screenshot Computer Stimulation

Figure 1 shows the screenshot of the transmission line characteristic calculation application. In this application, users are asked to enter transmission line parameters, namely, Resistance (R), Inductance (L), Conduction (G), Capacitance (C), Frequency (f), Transmission Line Length (1), and Load Impedance $\left.\left(\mathrm{Z} \_\mathrm{L}\right)\right)$. When the parameter values are entered, the application will automatically calculate and display the characteristics of the transmission channel in the form of Transmission Constant $(\gamma)$, Wavelength $(\lambda)$, Phase Speed (v), Characteristic Impedance (Z_O), Reflection Coefficient $(\Gamma)$, Transmission Coefficient $(\tau)$, Standing Wave Ratio (s), and Input Impedance (Z_in). The characteristics of the transmission line are calculated using the following equation

a. Constant propagation $(\gamma)$

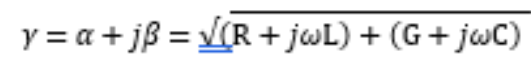

b. Wavelength $(\lambda)$

$$
n-\frac{2 \pi}{\beta}
$$

c. Phase Velocity $(v)$

$$
v=\begin{aligned}
& \omega \\
& \beta
\end{aligned}
$$

with $\omega=2 \pi f$

d. Characteristic Impedance $\left(\mathrm{Z}_{0}\right)$

$$
\mathrm{z}_{0}=\sqrt{\frac{\mathrm{R}+j \omega \mathrm{L}}{\mathrm{G}+j \omega \mathrm{C}}}
$$

e. Reflection Coefficient $(\Gamma)$

f. Transmission Coefficient $(\tau)$

$$
I=\frac{\underline{\underline{Z_{1}}-\mathrm{Z}_{0}}}{\underline{\underline{\mathrm{Z}_{1}}} \mathrm{Z}_{0}}
$$

$$
\imath=\frac{2 Z_{\mathrm{L}}}{\mathrm{Z}_{\mathrm{L}}+\mathrm{Z}_{0}}
$$




\section{g. Standing Wave Ratio (s)}

$$
s=\begin{aligned}
& 1+|\Gamma| \\
& 1-|\Gamma|
\end{aligned}
$$

h. Input Impedance $\left(Z_{\text {in }}\right)$

$$
Z_{\text {in }}=Z_{o} \frac{Z_{L}+j Z_{0} \tan \beta_{1} l}{Z_{0}+z_{L} \tan \beta_{1} l}
$$

The process of creating an application begins with creating the syntax of an application without a GUI (graphical user interface). In creating syntax, a list of input variables is collected first. As previously explained, there are 6 input variables, namely resistance $(\mathrm{R})$, inductance $(\mathrm{L})$, conductance $(\mathrm{G})$, capacitance $(\mathrm{C})$, frequency (f), transmission line length (l), and load impedance (Z_L).

After all the input variables are calculated, then create a syntax to calculate the values of the output variables. By using MATLAB, it is very easy to write the output variable syntax. We just need to type the equation in MATLAB syntax. As for the previous equation, here is the syntax for calculating the output variable of the designed application.

\section{RESULTS AND DISCUSSION}

Evaluation of the use of learning media with computer simulations is carried out using an online system. all students who take this lecture are 25 students. In Figure 2, you can see the student learning styles Based on the data obtained, $68 \%$ of students have a visual learning style and the remaining $24 \%$ have an audio learning style and $8 \%$ have a kinetic learning style

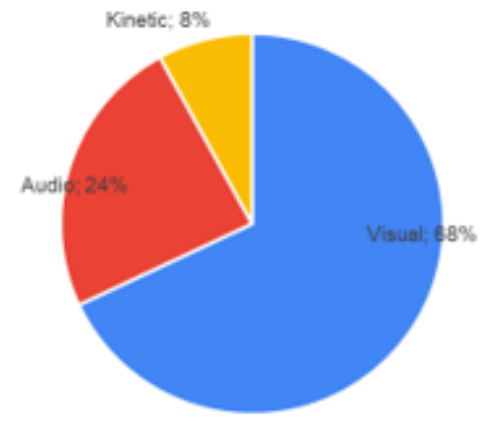

Figure 2. Student Style Study

Student responses obtained at the end of the lecture showed that the use of computer simulations was preferred by $52 \%$ of students. It is in accordance with

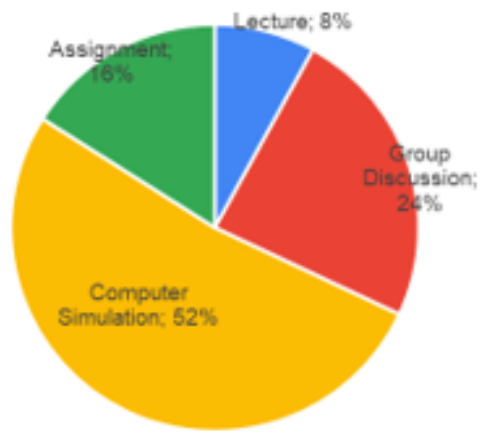

Figure 3. Learning Activity Student Preference

student learning styles dominated by visual learning styles. The overall results of the data can be seen in Figure 3.

In Figure 4, You can see student responses regarding the use of computer stimulations in lecture. The questions given consist of: (a) Stimulation is fun, (b) Better understand using simulation, (c) Simulation interface is good, and (d) Simulation easy to use.

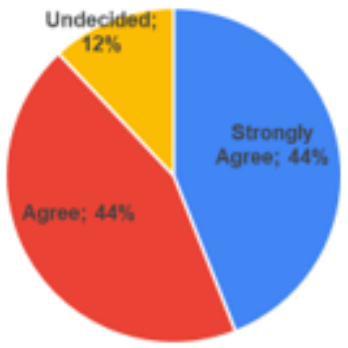

(a) Simulation is Fun

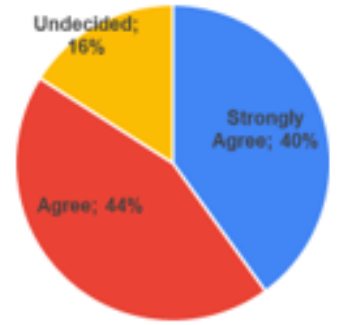

(b) Better understand using simulation

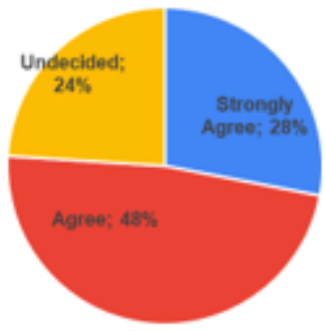

(c) Simulation interface is good 


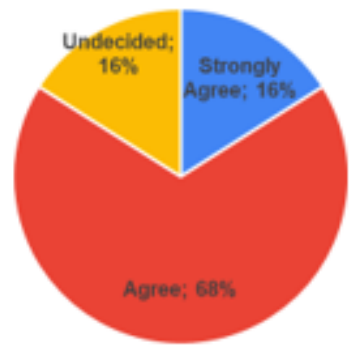

(d) Simulation easy to use

978-989-758-392-6, pages 156-159. DOI: $10.5220 / 0008682001560159$

[5] Yanuar, F.; Yozza, H.; Rahmi Hg, I. and Husna, R. (2018). Development of Learning Methods on Elementery Statistics Course Through Participatory Online Methods.In Improving Educational Quality Toward International Standard - Volume 1: ICEDQA, ISBN 978-989-758-392-6, pages 168-172. DOI: $10.5220 / 0008682401680172$

Figure 4. Student responses to the questionnaire.

\section{CONCLUSION}

Computer simulations designed to improve student learning activities are well accepted. based on student responses to learning activities that are preferred, the use of computer simulations is $52 \%$, then group discussions, assignments and lectures are 24\%, 16\% and $8 \%$ respectively

\section{ACKNOWLEDGMENT}

This work wass upported by Lembaga Pengembangan Pembelajaran dan Penjaminan Mutu Universitas Andalas. Contract \# T.03/UN.16.18/PT.01.03/2020.

\section{REFERENCES}

[1] Yulianti, L. and Syafruddin, (2018). The Application of Discovery Learning Method and Small Group Discussion in PAM - 472 Topics in Combinatorial Mathematics II.In Improving Educational Quality Toward International Standard Volume 1: ICED-QA, ISBN 978-989-758-392-6, pages 57-63. DOI: 10.5220/0008679400570063

[2] Nurkanti, M.; Utari, T. and Devi, C. (2018). Improve Student Learning Outcomes Through the Use of Interactive Visual Learning Media (MIVI). In Improving Educational Quality Toward International Standard -Volume 1: ICED-QA, ISBN 978-989-758392-6, pages 64-68. DOI: $10.5220 / 0008679500640068$

[3] Marnita AS, R. and Faradika, V. (2018). Using Weblog to Enhance Students' Motivation and Engagement in the Introduction to Journalism Class.In Improving Educational Quality Toward International

[4] Yuniza, A. and Mahata, M. (2018). Students' Responses to The Improvements of Learning and Practicum Services on Poultry Nutrition Science Subject.In Improving Educational Quality Toward International Standard - Volume 1: ICED-QA, ISBN 